\title{
Article \\ Biomechanical Analysis of Running in Shoes with Different Heel-to-Toe Drops
}

\author{
Masen Zhang ${ }^{1}$, Huijuan Shi ${ }^{1}$, Hui Liu ${ }^{2}$ and Xinglong Zhou ${ }^{1, *}$ \\ 1 Biomechanics Laboratory, School of Sport Science, Beijing Sport University, Beijing 100084, China; \\ zhangmasenvip@163.com (M.Z.); shihuijuan1103@163.com (H.S.) \\ 2 China Institute of Sport and Health Science, Beijing Sport University, Beijing 100084, China; \\ liuhuibupe@163.com \\ * Correspondence: zhouxinglong@bsu.edu.cn; Tel.: +86-130-0108-8208
}

check for updates

Citation: Zhang, M.; Shi, H.; Liu, H.; Zhou, X. Biomechanical Analysis of Running in Shoes with Different Heel-to-Toe Drops. Appl. Sci. 2021, 11 12144. https://doi.org/10.3390/ app112412144

Academic Editors: Roozbeh Naemi and Andrzej Wit

Received: 3 November 2021

Accepted: 10 December 2021

Published: 20 December 2021

Publisher's Note: MDPI stays neutral with regard to jurisdictional claims in published maps and institutional affiliations.

Copyright: (c) 2021 by the authors. Licensee MDPI, Basel, Switzerland. This article is an open access article distributed under the terms and conditions of the Creative Commons Attribution (CC BY) license (https:// creativecommons.org/licenses/by/ $4.0 /)$.

\begin{abstract}
The heel-to-toe drop of running shoes is a key parameter influencing lower extremity kinematics during running. Previous studies testing running shoes with lower or larger drops generally used minimalist or maximalist shoes, where the factors outside of the drop may lead to the observed changes in running biomechanics. Therefore, our aim was to compare the strike patterns, impact force, and lower extremity biomechanics when running in shoes that varied only in their drops. Eighteen habitual rearfoot strikers performed trials wearing running shoes with four drop conditions: $15 \mathrm{~mm}, 10 \mathrm{~mm}, 5 \mathrm{~mm}$, and without a drop. Three-dimensional (3D) tracks of the reflective markers and impact force were synchronously collected using a video graphic acquisition system and two force plates. The biomechanical parameters were compared among the four drop conditions using one-way ANOVA of repeated measures. A greater foot inclination angle $\left(p=0.001, \eta \mathrm{p}^{2}=0.36\right)$ at initial contact and a lower vertical loading rate $\left(p=0.002, \eta \mathrm{p}^{2}=0.32\right)$ during the standing phase were found when running in shoes with large drops compared with running in shoes without a drop. Running in shoes with large drops, as opposed to without, significantly increased the peak knee extension moment $\left(p=0.002, \eta p^{2}=0.27\right)$, but decreased the peak ankle eversion moment $\left(p=0.001, \eta \mathrm{p}^{2}=0.35\right.$ ). These findings suggest that the heel-to-toe drop of running shoes significantly influences the running pattern and the loading on lower extremity joints. Running shoes with large drops may be disadvantageous for runners with knee weakness and advantageous for runners with ankle weakness.
\end{abstract}

Keywords: heel-to-toe drop; strike pattern; knee; ankle; injury

\section{Introduction}

As one of the most popular sporting activities, running is an effective way to improve cardiovascular function and physical fitness [1]. However, running-related injuries are very common. A study has shown that $79 \%$ of long-distance runners suffer runningrelated injuries every year [2], such as patellofemoral pain, tibial stress fractures, Achilles tendonitis, and plantar fasciitis, with $46 \%$ of these injuries being recurrent. As the key equipment for running activity, well- designed running shoes are very important for optimizing the impact force and lower extremity loading that are related to running injuries. In spite of the constant innovation in running shoe design and advances in cushioning technology in the past few decades, running-related injuries and recurrence rates remain surprisingly high [3].

Various factors may lead to the etiology of running-related injuries. The amount, frequency, and timing of vertical impact force are considered to be the key factors causing lower extremity running-related injuries [4-6]. Vertical impact force can be affected by a few factors, including running shoes, foot strike patterns, and spatiotemporal parameters such as step length and frequency [7-11]. Therefore, mitigating the factors associated 
with vertical impact force seems to be the focus when aiming to decrease the risk of running-related injuries.

In order to decrease the vertical impact force, running shoe companies have launched higher heel-cushioned shoes, hoping to provide better cushioning and reduce more shocks due to impact force. A study revealed that additional cushioning reduced the vertical loading rate in running [12]. In recent years, some shoe companies altered the amount of cushioning in their shoes, offering running shoes with different drops (i.e., the thickness difference between the forefoot and heel of running shoes). Minimalist shoes are the representatives of shoes with lower drops which aim to simulate barefoot running and may promote non-rearfoot strike patterns. Studies have shown that running in minimalist shoes increased the vertical impact and vertical loading rate [8,13-15]. In addition, some studies have found that the cushion of running shoes has no immediate effect on the vertical impact force or vertical loading rate $[5,16]$. The conflict in previous studies' results may be caused by the differences in running speed, running environment, and limited range of drops. More importantly, previous studies used different brands and models of running shoes which had different midsole construction designs and midsole materials. Thus, it is unclear whether the observed different biomechanics during running in different types of shoes results from the drop or other structural aspects of running shoes.

Only a few studies have specifically focused on the drop of running shoes, which was reported to be a key parameter affecting the strike patterns. Studies have reported that an increased drop in running shoes promotes a rearfoot strike running gait [17,18]. Running in shoes with large drops increases the foot inclination angle at initial contact and decreases the vertical loading rate in the standing phase compared with running in shoes without a drop for female runners [7]. In addition to the biomechanical changes, a prospective study indicated that running in shoes with a 10-mm drop yields a higher injury rate for the knee joint and lower injury rate for the ankle and foot compared with running in shoes without a drop [19]. Few studies have focused on the biomechanical analysis of running shoes with various drops, which limits the understanding of the relationship between the drop of running shoes and lower extremity biomechanics.

The purpose of the current study was to compare the strike patterns, vertical impact forces, and lower extremity biomechanics during running in shoes that varied only in terms of heel-to-toe drop. We hypothesized that the foot inclination angle at initial contact would be greater when running in shoes with large drops (e.g., $15 \mathrm{~mm}$ and $10 \mathrm{~mm}$ ) compared with running in shoes without a drop. We also hypothesized that the vertical loading rate would be lower when running in shoes with large drops compared with running in shoes without a drop. We further hypothesized that the peak knee extension moment would be greater when running in shoes with large drops compared with running in shoes without a drop. Lastly, we hypothesized that the peak ankle plantarflexion moment would be lower when running in shoes with large drops compared with running in shoes without a drop.

\section{Materials and Methods}

\subsection{Participants}

Eighteen rearfoot strikers (mean age $=23.1 \pm 1.6$ years; mean standing height $=1.75 \pm 0.05 \mathrm{~m}$; mean body mass $=65.0 \pm 3.9 \mathrm{~kg}$ ) who participated in running activities at least 3 times per week and $20 \mathrm{~km}$ a week volunteered to participate in the current study. Participants were excluded from this study if they were unable to complete the test due to any musculoskeletal or nervous disorders or injuries in the 6 months prior to the study. Written informed consent was obtained from each participant, and the protocol for human subjects was approved by the Biomedical Internal Review Committee of Beijing Sport University.

\subsection{Experimental Protocol}

Each participant warmed up at their preferred speed on a treadmill for $10 \mathrm{~min}$ and then wore the same style of tight clothing. Nineteen retroreflective markers were then placed at the anterior superior iliac spines, lateral thighs, medial and lateral femoral 
condyles, anterior superior shanks, and medial and lateral malleoli, posterior calcaneus, and centers of the second and third metatarsals. An additional marker was placed between the fourth and fifth lumbar vertebrae. The markers at the medial femoral condyles and medial malleoli were removed after a standing static calibration test.

The participant was instructed to run in shoes with drops at $15 \mathrm{~mm}, 10 \mathrm{~mm}$, and $5 \mathrm{~mm}$, and without a drop (Figure 1), referred to in the current study as the shoe condition, in a random order. All running shoes had identical appearances and structures and were made from the same material with a forefoot part $12 \mathrm{~mm}$ in thickness. In each running test, the participant was instructed to run on a $20-\mathrm{m}$ runway at $4.0 \pm 0.2 \mathrm{~m} / \mathrm{s}$ in each shoe condition. Each participant completed three successful tests for each shoe. A successful test was defined as a test in which the participants had no less than six steps before and after the entire right foot stepped on a force plate without any step length adjustment.

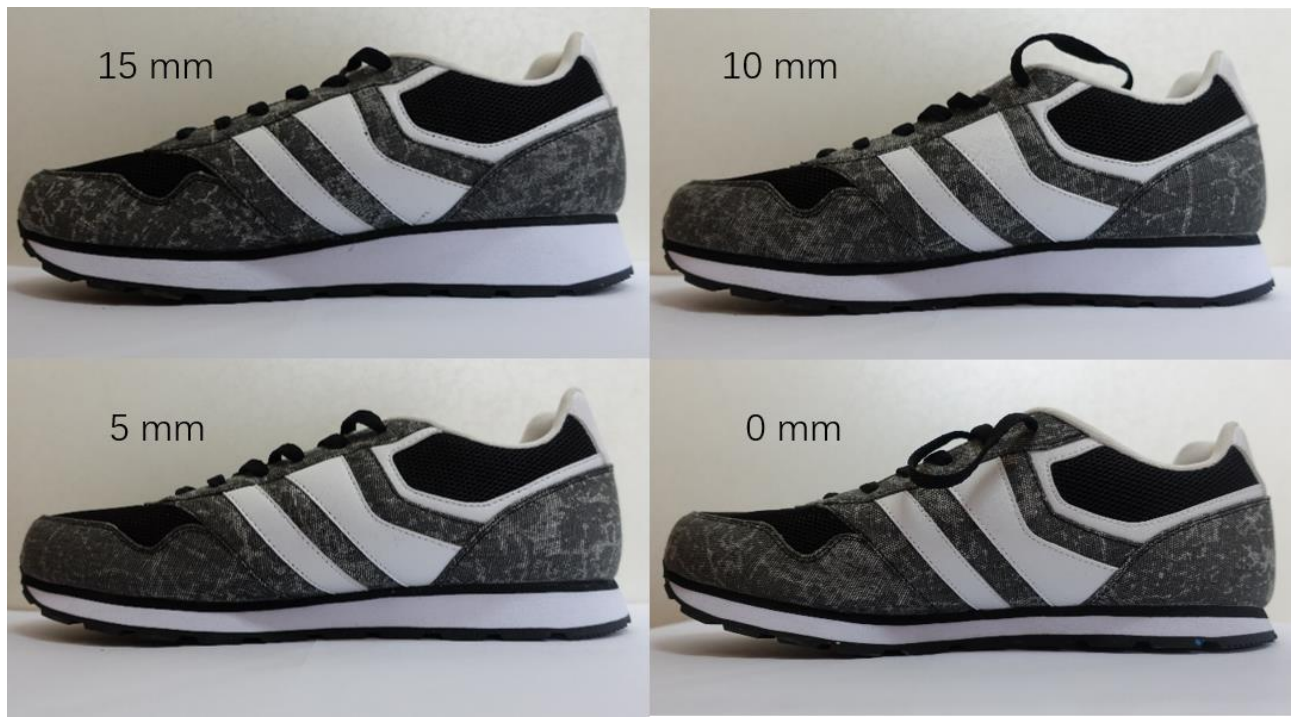

Figure 1. Schematic diagram of running shoes with $15 \mathrm{~mm}, 10 \mathrm{~mm}, 5 \mathrm{~mm}$, and no drop.

\subsection{Data Collection}

The running speed of the participants was monitored using two timing gates (Brower Timing Systems, Salt Lake City, UT, USA) placed on both sides of the force plates with a spacing of $3 \mathrm{~m}$. Three-dimensional (3D) tracks of reflective markers in the running tests were obtained using a video graphic acquisition system with eight cameras (Motion Analysis Corporation, Raptor-4, Santa Rosa, CA, USA) at $200 \mathrm{~Hz}$. Impact force data were obtained using 4 force plates (Kistler, Winterhur, Switzerland) buried in the middle of the runway at $1000 \mathrm{~Hz}$. The marker tracks and impact force data collections were timesynchronized with the Motion Analysis computer program package.

\subsection{Data Reduction}

The 3D coordinate tracks of the markers were filtered by a Butterworth low-pass digital filter with frequency of $12 \mathrm{~Hz}$ [20]. The definitions of the hip-, knee- and ankle-joint center and three-dimensional angle of the knee and ankle joints were consistent with the literature [21,22]. The vertical impact force of $20 \mathrm{~N}$ was defined as the threshold for determining the initial contact and toe-off time [23]. The standing phase was the duration between the initial contact and subsequent toe-off. The long axis of the foot was the line connecting the posterior calcaneus and the centers of the second and third metatarsals. The foot inclination angle was the angle between the long axis of the foot and the ground at initial contact. The angle was calculated by subtracting the angle during the standing static calibration from the angle of initial contact during running. The vertical impact peak and the vertical active peak were the first and second maximal values of the vertical impact force in the standing phase. The vertical loading rate was calculated by dividing 
the vertical impact peak by the duration between the initial contact and the time of the vertical impact peak. Joint moments were calculated using a standard inverse dynamics procedure [24].

\subsection{Statistical Analysis}

One-way ANOVA with repeated measures was performed to test our four hypotheses by determining the effects of the drop of running shoes on the foot inclination angle, vertical impact peak, vertical loading rate, vertical active peak, and lower extremity biomechanics with the global alpha at 0.05 . An effect size $\left(\eta_{p}{ }^{2}\right)$ of no less than 0.07 was considered to be a medium effect, while an effect size of no less than 0.14 was considered to be a large effect. When significant main effects were detected, post hoc paired $t$-tests were performed to locate differences between the drop conditions and no-drop condition with Bonferroni correction. A type I error rate no greater than 0.017 was considered as the index of statistical significance. All statistical procedures were conducted by use of version 20.0 of the SPSS Package (SPSS, Chicago, IL, USA).

\section{Results}

The average speed of running across all participants was $4.0 \pm 0.2 \mathrm{~m} / \mathrm{s}$. No significant differences in step length or step frequency were detected when wearing shoes with different drops $\left(p=0.088, \eta_{\mathrm{p}}{ }^{2}=0.12 ; p=0.563, \eta_{\mathrm{p}}{ }^{2}=0.04\right)($ Table 1$)$.

Table 1. Comparison of spatiotemporal parameters and impact force among the four shoe conditions.

\begin{tabular}{|c|c|c|c|c|}
\hline \multirow{2}{*}{ Variable } & \multicolumn{4}{|c|}{ Heel-to-Toe Drop (mm) } \\
\hline & 15 & 10 & 5 & 0 \\
\hline Running speed (m/s) & $4.0 \pm 0.1$ & $4.0 \pm 0.2$ & $4.0 \pm 0.2$ & $4.0 \pm 0.1$ \\
\hline Step length $(\mathrm{m})$ & $1.52 \pm 0.02$ & $1.50 \pm 0.03$ & $1.47 \pm 0.02$ & $1.47 \pm 0.02$ \\
\hline Step frequency (steps / min) & $163.2 \pm 7.5$ & $161.7 \pm 9.6$ & $164.4 \pm 10.4$ & $164.1 \pm 12.6$ \\
\hline $\begin{array}{l}\text { Foot inclination angle at } \\
\text { initial contact }\left(^{\circ}\right)\end{array}$ & $28.8 \pm 6.9 *$ & $28.3 \pm 7.6^{*}$ & $27.2 \pm 7.7^{*}$ & $22.6 \pm 8.5$ \\
\hline Vertical impact peak (BW) & $2.01 \pm 0.35$ & $2.03 \pm 0.35$ & $2.02 \pm 0.39$ & $2.05 \pm 0.40$ \\
\hline $\begin{array}{c}\text { Vertical loading rate } \\
\left(\mathrm{BW} \cdot \mathrm{s}^{-1}\right)\end{array}$ & $67.1 \pm 15.2 *$ & $68.9 \pm 12.5^{*}$ & $72.9 \pm 14.2$ & $85.2 \pm 19.1$ \\
\hline Vertical active peak (BW) & $2.73 \pm 0.32$ & $2.70 \pm 0.33$ & $2.69 \pm 0.28$ & $2.75 \pm 0.33$ \\
\hline
\end{tabular}

A significant main effect of the drop of running shoes on the foot inclination angle at initial contact was detected $\left(p=0.001, \eta_{\mathrm{p}}{ }^{2}=0.36\right)$ (Table 1$)$. Post hoc paired $t$-tests revealed that the foot inclination angle at initial contact was significantly greater when running in shoes with $15 \mathrm{~mm}, 10 \mathrm{~mm}$, and $5 \mathrm{~mm}$ drops compared with running in shoes without a $\operatorname{drop}(p=0.001 ; p=0.001 ; p=0.004)$. No significant differences in the knee flexion angle or ankle plantarflexion angle at initial contact were detected when running in shoes with different drops $\left(p=0.617, \eta_{\mathrm{p}}{ }^{2}=0.04 ; p=0.418, \eta_{\mathrm{p}}{ }^{2}=0.05\right)$ (Table 1$)$.

No significant differences in the vertical impact peak or the vertical active peak were detected $\left(p=0.933, \eta_{\mathrm{p}}{ }^{2}=0.01 ; p=0.506, \eta_{\mathrm{p}}{ }^{2}=0.04\right)$ (Table 1$)$. A significant main effect of the drop of running shoes on the vertical loading rate was detected $\left(p=0.002, \eta_{\mathrm{p}}{ }^{2}=0.32\right)$ (Table 1). Post hoc paired $t$-tests revealed that the vertical loading rate was significantly lower when running in shoes with $15-\mathrm{mm}$ and $10-\mathrm{mm}$ drops compared with running in shoes without a drop $(p=0.005 ; p=0.001)$. No significant difference in the vertical loading rate was detected between running in shoes with a 5-mm drop and without a drop $(p=0.030)$.

A significant main effect of the drop of running shoes on the peak knee flexion angle was detected $\left(p=0.007, \eta_{\mathrm{p}}{ }^{2}=0.27\right)$ (Table 2). Post hoc paired $t$-tests revealed that the peak knee flexion angle was greater when running in shoes with $15-\mathrm{mm}, 10-\mathrm{mm}$, and $5-\mathrm{mm}$ drops compared with running in shoes without a drop $(p=0.012 ; p=0.001 ; p=0.003)$. 
Table 2. Comparison of biomechanical data of the knee and ankle joints among the four shoe conditions.

\begin{tabular}{|c|c|c|c|c|}
\hline \multirow[b]{2}{*}{ Variable } & \multicolumn{4}{|c|}{ Heel-to-Toe Drop (mm) } \\
\hline & 15 & 10 & 5 & $\mathbf{0}$ \\
\hline \multicolumn{5}{|l|}{ Knee joint } \\
\hline $\begin{array}{l}\text { Flexion angle at initial } \\
\operatorname{contact}\left({ }^{\circ}\right)\end{array}$ & $16.4 \pm 7.1$ & $16.4 \pm 7.6$ & $16.5 \pm 6.1$ & $17.8 \pm 5.8$ \\
\hline Peak flexion angle $\left(^{\circ}\right)$ & $47.1 \pm 4.7^{*}$ & $47.2 \pm 4.6^{*}$ & $46.3 \pm 3.8 *$ & $44.5 \pm 4.2$ \\
\hline $\begin{array}{l}\text { Peak flexion moment } \\
\left(\mathrm{N} \cdot \mathrm{m} \cdot \mathrm{kg}^{-1}\right)\end{array}$ & $0.52 \pm 0.11$ & $0.50 \pm 0.19$ & $0.45 \pm 0.15$ & $0.49 \pm 0.16$ \\
\hline $\begin{array}{l}\text { Peak extension moment } \\
\left(\mathrm{N} \cdot \mathrm{m} \cdot \mathrm{kg}^{-1}\right)\end{array}$ & $2.98 \pm 0.51 *$ & $3.11 \pm 0.51 *$ & $2.91 \pm 0.54$ & $2.70 \pm 0.69$ \\
\hline Peak abduction angle $\left(^{\circ}\right)$ & $1.3 \pm 2.2$ & $0.8 \pm 2.6$ & $0.7 \pm 3.8$ & $2.0 \pm 3.3$ \\
\hline $\begin{array}{l}\text { Peak abduction moment } \\
\left(\mathrm{N} \cdot \mathrm{m} \cdot \mathrm{kg}^{-1}\right)\end{array}$ & $0.45 \pm 0.27$ & $0.51 \pm 0.27$ & $0.48 \pm 0.24$ & $0.55 \pm 0.22$ \\
\hline \multicolumn{5}{|l|}{ Ankle joint } \\
\hline $\begin{array}{l}\text { Plantarflexion angle at initial } \\
\text { contact }\left(^{\circ}\right)\end{array}$ & $2.1 \pm 6.3$ & $2.1 \pm 6.7$ & $2.5 \pm 6.4$ & $1.4 \pm 7.3$ \\
\hline Peak plantarflexion angle $\left({ }^{\circ}\right)$ & $33.9 \pm 4.8$ & $35.1 \pm 5.0$ & $34.5 \pm 3.9$ & $34.5 \pm 4.9$ \\
\hline $\begin{array}{l}\text { Peak plantarflexion moment } \\
\left(\mathrm{N} \cdot \mathrm{m} \cdot \mathrm{kg}^{-1}\right)\end{array}$ & $2.77 \pm 0.46$ & $2.71 \pm 0.48$ & $2.75 \pm 0.38$ & $2.85 \pm 0.44$ \\
\hline $\begin{array}{l}\text { Peak dorsiflexion moment } \\
\left(\mathrm{N} \cdot \mathrm{m} \cdot \mathrm{kg}^{-1}\right)\end{array}$ & $0.22 \pm 0.12$ & $0.27 \pm 0.12$ & $0.25 \pm 0.12$ & $0.21 \pm 0.12$ \\
\hline Peak eversion angle $\left(^{\circ}\right)$ & $0.2 \pm 2.7 *$ & $0.7 \pm 3.0$ * & $1.2 \pm 3.0$ & $3.0 \pm 2.8$ \\
\hline $\begin{array}{l}\text { Peak eversion moment } \\
\left(\mathrm{N} \cdot \mathrm{m} \cdot \mathrm{kg}^{-1}\right)\end{array}$ & $0.41 \pm 0.20$ * & $0.48 \pm 0.14$ * & $0.57 \pm 0.17$ & $0.64 \pm 0.32$ \\
\hline
\end{tabular}

Note: BW = body weight. ${ }^{*} p<0.017$, significantly different from no heel-to-toe drop.

A significant main effect of the drop of running shoes on the peak knee extension moment was detected $\left(p=0.002, \eta_{\mathrm{p}}{ }^{2}=0.27\right)$ (Table 2). Post hoc paired $t$-tests revealed that the peak knee extension moment was greater when running in shoes with $15-\mathrm{mm}$ and $10-\mathrm{mm}$ drops compared with running in shoes without a drop $(p=0.015 ; p=0.003)$. No significant difference in the peak knee extension moment was detected between the running shoes with a 5-mm drop and those without a drop $(p=0.072)$.

A significant main effect of the drop of running shoes on the peak ankle eversion angle was detected $\left(p=0.002, \eta_{\mathrm{p}}{ }^{2}=0.29\right)$ (Table 2). Post hoc paired $t$-tests revealed that the peak ankle eversion angle was significantly lower when running in shoes with $15-\mathrm{mm}$ and $10-\mathrm{mm}$ drops compared with running in shoes without a drop $(p=0.004 ; p=0.006)$. No significant difference in the peak ankle eversion angle was detected between the running shoes with a 5-mm drop and those without a drop $(p=0.018)$.

No main effect of the drop of running shoes on the peak ankle plantarflexion moment was detected $\left(p=0.152, \eta_{\mathrm{p}}{ }^{2}=0.10\right)$ (Table 2). A significant main effect of the drop of running shoes on the peak ankle eversion moment was detected $\left(p=0.001, \eta_{\mathrm{p}}{ }^{2}=0.35\right)$ (Table 2). Post hoc paired $t$-tests revealed that the peak ankle eversion moment was significantly lower when running in shoes with 15-mm and 10-mm drops compared with running in shoes without a drop $(p=0.004 ; p=0.005)$. No significant difference in the peak ankle eversion moment was detected between the running shoes with a 5-mm drop and those without a drop $(p=0.167)$.

\section{Discussion}

In the current study, the participants were tested in running shoes that varied only in terms of heel-to-toe drop, and the average running speed across all the participants was $4.0 \pm 0.2 \mathrm{~m} / \mathrm{s}$. Running in shoes with large drops increased the foot inclination angle at initial contact, decreased the vertical loading rate, increased the peak knee flexion angle and extension moment, and decreased the peak ankle eversion angle and moment during the standing phase. Thus, the heel-to-toe drop of the running shoes was a key factor influencing the running pattern and the loading of the lower extremity joints. 
The results of the current study support our first hypothesis that the foot inclination angle at initial contact would be greater when running in shoes with large drops compared with running in shoes without a drop. Our results revealed that the foot inclination angle at initial contact was more than $5.7^{\circ}$ larger when running in shoes with large drops compared with running in shoes without a drop. No significant differences in the knee flexion angle or ankle plantarflexion angle were detected at initial contact. The effect of shoes without a drop on the gait parameters was much less than that of minimalist shoes. Runners struck the ground with less ankle dorsiflexion and more knee flexion [8] while running in minimalist shoes, which may be explained by features of the minimalist shoes other than drop. A previous study also found a positive correlation between the drop and foot inclination angle at initial contact for rearfoot runners [17]. With the increase in the foot inclination angle at initial contact, the knee joint's function as an energy absorber was increased [23]. When running in shoes with large drops, runners attempted to contact the ground with the rearfoot at initial contact, which placed the supporting leg further beneath the center of the body mass, thus increasing the quadriceps muscles arm and the knee extension moments [24,25].

The results of the current study support our second hypothesis that the vertical loading rate would be lower when running in shoes with large drops compared with running in shoes without a drop. According to the traditional view, the cushioning structure of running shoes allows for more material deformation and lowers the impact force, thereby playing an important role in protection of the lower extremities during running. Another view is that running shoes with large drops would promote a rearfoot strike pattern that increases the impact force [26]. Several studies have shown mixed results for the effect of the drop of running shoes on the vertical impact force and the vertical loading rate $[5,8,13-15,27]$. This disagreement is likely caused by the use of different brands and models of running shoes, which have different midsole construction designs and midsole materials. The results in our study suggest that the observed differences in previous research among shoes with respect to the vertical impact force may have stemmed from factors other than drop, such as the midsole geometry, midsole stiffness, midsole thickness, or shoe weight, and that the drop of running shoes was a key factor influencing the vertical loading rate.

Our study compared running shoes that varied only in terms of the drop and showed that running in shoes with large drops did not change the vertical impact force but significantly decreased the vertical loading rate. Previous studies, in accordance with our study, have shown a decrease in the vertical loading rate when increasing the drop of running shoes or the foot inclination angle $[7,28]$. At initial contact and during the early standing phase, ankle dorsiflexion was significantly weakened when running in shoes without a drop, suggesting the pre-activation of the plantarflexion muscles was greater. The higher vertical loading rate observed in shoes without a drop was in accordance with previous studies that showed a significant positive correlation between the amplitude of pre-activation of the gastrocnemius and the vertical impact peak [7,29]. Combined with a previous study, the results of current study may have significance in preventing pathologies. The previous study showed that a higher body fat percentage, suggesting excessive loading and musculoskeletal pain, was associated with depression and poor health-related quality of life in an older population [30]. Considering that higher loading rates are associated with lower extremity injuries, it is recommended to wear running shoes with large drops to reduce lower extremity injuries, and this may improve the health-related quality of life.

The results of the current study support our third hypothesis that the peak knee extension moment would be greater when running in shoes with large drops compared with running in shoes without a drop. Our results showed a greater knee flexion angle and extension moment during the middle standing phase when wearing shoes with large drops but no significant differences for the knee flexion angle at initial contact, which is consistent with the conclusions of a previous study. Higher knee extension moments may indicate greater activity of the quadriceps muscles, which can increase the force transmitted to the patellar tendon, strain around the patella, and patellofemoral stress [7]. Patellofemoral 
pain is a very common lower limb injury for runners [2]. Elevated patellofemoral stress is proposed as the direct cause or mechanism of patellofemoral pain, and patients with patellofemoral pain exhibit greater knee extension moments and patellofemoral stress than the pain-free controls $[31,32]$. Our results suggest that running in shoes without a drop might minimize strain around the knee, which may have therapeutic benefits in reducing the risk of knee injuries and patellofemoral pain. Previous studies have demonstrated that barefoot running and running in minimalist shoes were associated with decreased knee moment and patellofemoral stress, respectively, compared with traditional running shoes. Thus, this may reduce a runner's risk of patellofemoral pain $[15,23]$. Running shoes without a drop may be a better choice, as they can not only reduce knee joint loading but also provide better protection for the foot compared with barefoot and minimalist shoes. Future studies should further determine the long-term influence of switching to running shoes without a drop on the biomechanics of the lower extremities.

The results of the current study do not support our fourth hypothesis that the peak ankle plantarflexion moment would be lower when running in shoes with large drops compared with running in shoes without a drop. However, lower peak ankle eversion angles and moments were found when running in shoes with 15-mm and 10-mm drops compared with running in shoes without a drop. Another study showed a significantly lower ankle plantarflexion moment during the braking phase between $12 \%$ and $40 \%$ of the standing phase for female runners when running in shoes with large drops [7]. Runners suffering from or predicted to develop Achilles tendon disease or medial tibial stress syndrome displayed prolonged ankle eversion durations and greater ankle eversion moments compared with the pain-free controls $[33,34]$. The increase in calf triceps muscle strain may be the reason for most anecdotal reports of calf and Achilles tendon pain during the first forefoot running or running in forefoot running shoes. In other words, running in shoes with large drops may minimize the net ankle moment during running and decrease the loading of the Achilles tendon and the calf triceps for runners prone to Achilles tendon disease and medial tibial stress syndrome.

Our results also provide evidence for the clinical observations that running in shoes with a 10-mm drop was associated with a higher rate of knee joint injuries and a lower rate of ankle and foot injuries compared with running in shoes without a drop. The previous study showed that the rate of knee joint injuries was $26 \%$ when running in shoes with a 10-mm drop, which was greater than that $(15 \%)$ when running in shoes without a drop, whereas the rate of ankle and foot injuries was $31 \%$ when running in shoes with a 10-mm drop, which was lower than that (41\%) when running in shoes without a drop [19]. This was mainly due to the magnitudes of knee and ankle joint loading. The results of our study revealed that the peak knee extension moment was $3.11 \pm 0.51 \mathrm{~N} \cdot \mathrm{m} \cdot \mathrm{kg}^{-1}$ when running in shoes with a 10-mm drop, which was $15 \%$ greater than the $2.70 \pm 0.69 \mathrm{~N} \cdot \mathrm{m} \cdot \mathrm{kg}^{-1}$ recorded when running in shoes without a drop. The results of the current study also revealed that the peak ankle eversion moment was $0.48 \pm 0.14 \mathrm{~N} \cdot \mathrm{m} \cdot \mathrm{kg}^{-1}$ when running in shoes with a 10-mm drop, which was $25 \%$ lower than the $0.64 \pm 0.32 \mathrm{~N} \cdot \mathrm{m} \cdot \mathrm{kg}^{-1}$ recorded when running in shoes without a drop. These results, combined with the results of the previous studies, indicate that wearing shoes with a 10-mm drop may be disadvantageous for runners with knee weakness and advantageous for runners with ankle weakness $[7,19]$. These findings can help runners, physicians, and running shoe designers better understand the potential mechanism of running-related injuries and contribute to the development of running shoes.

\section{Conclusions}

In conclusion, the heel-to-toe drop of running shoes can influence the running pattern and loading on the lower extremity joints. Running in shoes with large drops significantly increased the knee extension moment and decreased the ankle eversion moment during the standing phase. These findings suggest that running shoes with large drops may be disadvantageous for runners with knee weakness and advantageous for runners with ankle weakness. 
Author Contributions: Conceptualization, X.Z.; formal analysis, M.Z. and H.S.; funding acquisition, H.L.; investigation, H.S.; methodology, X.Z.; project administration, H.L.; resources, H.L.; supervision, X.Z.; writing-original draft, M.Z. All authors have read and agreed to the published version of the manuscript.

Funding: This study was supported by the National Natural Science Foundation of China (grant number: 81572212).

Institutional Review Board Statement: The study was conducted according to the guidelines of the Declaration of Helsinki and approved by the Ethics Committee of Beijing Sport University (protocol code $2020133 \mathrm{H})$.

Informed Consent Statement: Informed consent was obtained from all subjects participating in current study.

Data Availability Statement: The data of the current study are available on reasonable request from the corresponding author.

Conflicts of Interest: The authors declare no conflict of interest.

\section{References}

1. Lee, D.C.; Pate, R.R.; Lavie, C.J.; Sui, X.; Church, T.S.; Blair, S.N. Leisure-time running reduces all-cause and cardiovascular mortality risk. J. Am. Coll. Cardiol. 2014, 64, 472-481. [CrossRef] [PubMed]

2. Van Gent, R.N.; Siem, D.; Van Middelkoop, M.; van Os, A.G.; Bierma-Zeinstra, S.M.A.; Koes, B.W. Incidence and determinants of lower extremity running injuries in long distance runners: A systematic review. Br. J. Sports Med. 2007, 41, 469-480. [CrossRef]

3. Lopes, A.D.; Hespanhol, L.C.; Yeung, S.S.; Costa, L.O.P. What are the Main Running-Related Musculoskeletal Injuries? Sport Med. 2012, 42, 891-905. [CrossRef] [PubMed]

4. Clarke, T.E.; Frederick, E.C.; Cooper, L.B. Effects of shoe cushioning upon ground reaction forces in running. Int. J. Sports Med. 1983, 4, 247-251. [CrossRef] [PubMed]

5. Aminaka, N.; Arthur, K. No immediate effects of highly cushioned shoes on basic running biomechanics. Kinesiology 2018, 50, 124-130. [CrossRef]

6. Altman, A.R.; Davis, I.S. Barefoot running: Biomechanics and implications for running injuries. Curr. Sports Med. Rep. 2012, 11, 244-250. [CrossRef] [PubMed]

7. Besson, T.; Morio, C.; Millet, G.Y.; Rossi, J. Influence of shoe drop on running kinematics and kinetics in female runners. Eur. J. Sport Sci. 2019, 19, 1320-1327. [CrossRef] [PubMed]

8. Hannigan, J.J.; Pollard, C.D. Differences in running biomechanics between a maximal, traditional, and minimal running shoe. J. Sci. Med. Sport 2020, 23, 15-19. [CrossRef] [PubMed]

9. Law, M.H.C.; Choi, E.M.F.; Law, S.H.Y.; Chan, S.S.C.; Wong, S.M.S.; Ching, E.C.K.; Chan, Z.Y.S.; Zhang, J.H.; Lam, G.W.K.; Lau, F.O.Y.; et al. Effects of footwear midsole thickness on running biomechanics. J. Sports Sci. 2019, 37, 1004-1010. [CrossRef]

10. Santos, A.F.; Nakagawa, T.H.; Serrao, F.V.; Ferber, R. Patellofemoral joint stress measured across three different running techniques. Gait Posture 2019, 68, 37-43. [CrossRef]

11. Heiderscheit, B.C.; Chumanov, E.S.; Michalski, M.P.; Wille, C.M.; Ryan, M.B. Effects of step rate manipulation on joint mechanics during running. Med. Sci. Sports Exerc. 2011, 43, 296-302. [CrossRef] [PubMed]

12. OLeary, K.; Vorpahl, K.; Heiderscheit, B. Effect of cushioned insoles on impact forces during running. J. Am. Podiatr. Med Assoc. 2008, 98, 36-41. [CrossRef] [PubMed]

13. Rice, H.; Patel, M. Manipulation of foot strike and footwear increases Achilles tendon loading during running. Am. J. Sports Med. 2017, 45, 2411-2417. [CrossRef] [PubMed]

14. Willy, R.; Davis, I.S. Kinematic and kinetic comparison of running in standard and minimalist shoes. Med. Sci. Sports Exerc. 2014, 46, 318-323. [CrossRef] [PubMed]

15. Sinclair, J.; Fau-Goodwin, J.; Richards, J.; Shore, H. The influence of minimalist and maximalist footwear on the kinetics and kinematics of running. Footwear Sci. 2016, 8, 33-39. [CrossRef]

16. Yang, C.; Xiao, S.; Yang, Y.; Zhang, X.; Wang, J.; Fu, W. Patellofemoral joint loads during running immediately changed by shoes with different minimalist indices: A cross-sectional study. Appl. Sci. 2019, 9, 4176. [CrossRef]

17. Horvais, N.; Samozino, P. Effect of midsole geometry on foot-strike pattern and running kinematics. Footwear Sci. 2013, 5, 81-89. [CrossRef]

18. Chambon, N.; Delattre, N.; Gueguen, N.; Berton, E.; Rao, G. Shoe drop has opposite influence on running pattern when running overground or on a treadmill. Eur. J. Appl. Physiol. 2015, 115, 911-918. [CrossRef]

19. Malisoux, L.; Chambon, N.; Urhausen, A.; Theisen, D. Influence of the heel-to-toe drop of standard cushioned running shoes on injury risk in leisure-time runners: A randomized controlled trial with 6-month follow-up. Am. J. Sports Med. 2016, 44, 2933-2940. [CrossRef] 
20. Nigg, B.; Hintzen, S.; Ferber, R. Effect of an unstable shoe construction on lower extremity gait characteristics. Clin. Biomech. 2006, 21, 82-88. [CrossRef]

21. Bell, A.L.; Pedersen, D.R.; Brand, R.A. A comparison of the accuracy of several hip center location prediction methods. J. Biomech. 1990, 23, 617-621. [CrossRef]

22. Zhang, L.; Li, H.; Garrett, W.E.; Liu, H.; Yu, B. Hamstring muscle-tendon unit lengthening and activation in instep and cut-off kicking. J. Biomech. 2019, 99, 109-114. [CrossRef] [PubMed]

23. Sinclair, J. Effects of barefoot and barefoot inspired footwear on knee and ankle loading during running. Clin. Biomech. 2014, 29, 395-399. [CrossRef]

24. Bonacci, J.; Vicenzino, B.; Spratford, W.; Collins, P. Take your shoes off to reduce patellofemoral joint stress during running. Br. J. Sports Med. 2014, 48, 425-428. [CrossRef] [PubMed]

25. Bonacci, J.; Saunders, P.U.; Hicks, A.; Rantalainen, T.; Vicenzino, B.; Guglielmo, T.; Spratford, W. Running in a minimalist and lightweight shoe is not the same as running barefoot: A biomechanical study. Br. J. Sports Med. 2013, 47, 387-392. [CrossRef]

26. Kulmala, J.-P.; Avela, J.; Pasanen, K.; Parkkari, J. Forefoot strikers exhibit lower running-induced knee loading than rearfoot strikers. Med. Sci. Sports Exerc. 2013, 45, 2306-2313. [CrossRef] [PubMed]

27. Chambon, N.; Delattre, N.; Guéguen, N.; Berton, E.; Rao, G. Is midsole thickness a key parameter for the running pattern? Gait Posture 2014, 40, 58-63. [CrossRef]

28. Breine, B.; Malcolm, P.; Van Caekenberghe, I.; Fiers, P.; Frederick, E.C.; De Clercq, D. Initial foot contact and related kinematics affect impact loading rate in running. J. Sports Sci. 2017, 35, 1556-1564. [CrossRef] [PubMed]

29. Giandolini, M.; Arnal, P.J.; Millet, G.Y.; Peyrot, N.; Samozino, P.; Dubois, B.; Morin, J.-B. Impact reduction during running: Efficiency of simple acute interventions in recreational runners. Eur. J. Appl. Physiol. Occup. Physiol. 2013, 113, 599-609. [CrossRef] [PubMed]

30. Giovannini, S.; Macchi, C.; Liperoti, R.; Laudisio, A.; Coraci, D.; Loreti, C.; Vannetti, F.; Onder, G.; Padua, L. Association of body fat with health-related quality of life and depression in nonagenarians: The mugello study. J. Am. Med Dir. Assoc. 2019, 20, 564-568. [CrossRef] [PubMed]

31. Witvrouw, E.; Callaghan, M.J.; Stefanik, J.J.; Noehren, B.; Bazett-Jones, D.M.; Willson, J.D.; Earl-Boehm, J.E.; Davis, I.S.; Powers, C.M.; McConnell, J.; et al. Patellofemoral pain: Consensus statement from the 3rd International Patellofemoral Pain Research Retreat held in Vancouver, September 2013. Br. J. Sports Med. 2014, 48, 411-414. [CrossRef] [PubMed]

32. Brechter, J.H.; Powers, C.M. Patellofemoral stress during walking in persons with and without patellofemoral pain. Med. Sci. Sports Exercise 2002, 34, 1582-1593. [CrossRef] [PubMed]

33. Becker, J.; James, S.; Wayner, R.; Osternig, L.; Chou, L.-S. Biomechanical factors associated with Achilles tendinopathy and medial tibial stress syndrome in runners. Am. J. Sports Med. 2017, 45, 2614-2621. [CrossRef] [PubMed]

34. Becker, J.; Nakajima, M.; Wu, W.F.W. Factors Contributing to Medial Tibial Stress Syndrome in Runners: A Prospective Study. Med. Sci. Sports Exerc. 2018, 50, 2092-2100. [CrossRef] [PubMed] 